\title{
Kotani Theory for One Dimensional Stochastic Jacobi Matrices ${ }^{\star}$
}

\section{Barry Simon}

Departments of Mathematics and Physics, California Institute of Technology, Pasadena, CA 91125, USA

\begin{abstract}
We consider families of operators, $H_{\omega}$, on $\ell_{2}$ given by $\left(H_{\omega} u\right)(n)=$ $u(n+1)+u(n-1)+V_{\omega}(n) u(n)$, where $V_{\omega}$ is a stationary bounded ergodic sequence. We prove analogs of Kotani's results, including that for a.e. $\omega, \sigma_{\mathrm{ac}}\left(H_{\omega}\right)$ is the essential closure of the set of $E$ where $\gamma(E)$ the Lyaponov index, vanishes and the result that if $V_{\omega}$ is non-deterministic, then $\sigma_{\mathrm{ac}}$ is empty.
\end{abstract}

\section{Introduction}

In a beautiful paper, Kotani [10] has proved three remarkable theorems about onedimensional stochastic Schrödinger operators, i.e. operators of the form $-d^{2} / d x^{2}+$ $V_{\omega}(x)$ on $L^{2}(-\infty, \infty)$, where $V_{\omega}$ is a stationary bounded ergodic process. It is not completely straightforward to extend his proofs to the case where $-d^{2} / d x^{2}$ is replaced by a finite difference operator, and that is our goal in this note.

Explicitly, let $(\Omega, \mu)$ be a probability measure space, $T$ a measure preserving invertible ergodic transformation, and $f$ a bounded measurable real-valued function. We define $V_{\omega}(n)=f\left(T^{n} \omega\right)$. We let $H_{\omega}$ be the operator on $\ell^{2}(Z)$

$$
\left(H_{\omega} u\right)(n)=u(n+1)+u(n-1)+V_{\omega}(n) u(n) .
$$

Integrals over $\omega$ will be denoted by $E(\cdot)$.

Given a subset, $J$, of $Z$, we let $\Sigma_{J}$ be the sigma-algebra generated by $\left\{V_{\omega}(n)\right\}_{n \in J}$. We say that the process is deterministic if $\Sigma_{-\infty} \equiv \bigcap_{j=1}^{\infty} \Sigma_{(-\infty,-j)}$ is up to sets of measure zero, $\Sigma_{(-\infty, \infty)}$; equivalently if $V_{\omega}(n)$ is a.e., a measurable function of $\left\{V_{\omega}(n)\right\}_{n \leqq 0}$. Otherwise it is non-deterministic. Almost periodic sequences are deterministic. Independent, identically distributed random variables are non-deterministic.

The Lyaponov index $\gamma(E)$ is defined, for example, in $[1,4]$. It can be characterized as follows: For each complex $E$, for a.e. $\omega$, any solution of $H_{\omega} u=E u$ (in sequence sense) has $\lim _{n \rightarrow \infty} \frac{1}{n} \ln \left[|u(n)|^{2}+|u(n+1)|^{2}\right]^{1 / 2}$ exists and it is either $\gamma$ or $-\gamma$. It is an

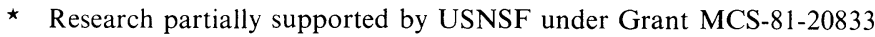


old result of Pastur [11] and Ishii [7] (see also Casher-Lebowitz [3]) that $\gamma(E)$ on the real axis is related to absolutely continuous spectrum.

Theorem $0([7,11])$. If $\gamma(E)>0$ on some set $A$ in $R$, then $E_{\omega}^{\mathrm{ac}}(A)=0$ for a.e. $\omega$, where $E_{\omega}^{\mathrm{ac}}$ is the absolute component of the spectral projection for $H_{\omega}$.

Here we will prove the following:

Theorem 1. If $\gamma(E)=0$ on a subset, $A$, of $R$ with positive Lebesgue measure, then $E_{\omega}^{\mathrm{ac}}(A) \neq 0$ for a.e. $\omega$.

Theorem 2. If $\gamma(E)=0$ on an open interval, $I$, of $R$, then for a.e. $\omega$, the spectral measures are purely absolutely continuous on $I$.

Theorem 3. If the hypotheses of Theorem 1 hold, then $V_{\omega}$ is deterministic.

Theorems 0 and 1 show that $\sigma_{\mathrm{ac}}$ is for a.e. $\omega$ the essential closure of the set where $\gamma(E)=0$. Theorem 3, which can be viewed as a kind of generalized Furstenberg theorem, says Thms. 1 and 2 aren't applicable very often. Theorems 0 and 3 imply that if $V$ is non-deterministic, $\sigma_{\mathrm{ac}}=\varnothing$. Theorems 1 and 2 are related to recent results of Carmona [2].

Theorems $I-3$ are precise analogs of the main results of Kotani [10] in the continuous case. Kotani uses functions $h_{ \pm}(\omega, E)$ defined for $\operatorname{Im} E>0$ by the following: If $\operatorname{Im} E>0$, there are unique (up to factor) solutions, $u_{ \pm}(x, \omega, E)$, of $-u^{\prime \prime}+(V-E) u=0$ which are $L^{2}$ at $\pm \infty$. Define

$$
h_{ \pm}(\omega, E)= \pm \frac{u_{ \pm}^{\prime}(0, \omega, E)}{u_{ \pm}(0, \omega, E)} .
$$

As is well-known, the Green's function obeys

$$
G^{\omega}(0,0 ; E)=-\left(h_{+}+h_{-}\right)^{-1} \text {. }
$$

Since $E(G)$ is the Borel transform of the density of states and the Thouless formula relates $\gamma$ to this density of states (see e.g. [1]), one has:

$$
E\left(\operatorname{Im}\left(\left[h_{+}+h_{-}\right]^{-1}\right)\right)=-\partial \gamma(E) / \partial(\operatorname{Im} E) .
$$

Using the formula of Johnson and Moser [8]

$$
E\left(\operatorname{Re} h_{+}\right)=E\left(\operatorname{Re} h_{-}\right)=-\gamma(E)
$$

Kotani then proves:

$$
E\left(\left(\operatorname{Im} h_{ \pm}\right)^{-1}\right)=2 \gamma(E) / \operatorname{Im} E .
$$

Equations (1.2) and (1.4) then imply

$$
\begin{aligned}
& E\left(\left[\left(\operatorname{Im} h_{+}\right)^{-1}+\left(\operatorname{Im} h_{-}\right)^{-1}\right]\left\{\left(\operatorname{Im} h_{+}-\operatorname{Im} h_{-}\right)^{2}+\left(\operatorname{Re} h_{+}+\operatorname{Re} h_{-}\right)^{2}\right\} /\left|h_{+}+h_{-}\right|^{2}\right) \\
& \quad=4\left[(\operatorname{Im} E)^{-1} \gamma(E)-\partial \gamma(E) / \partial \operatorname{Im} E\right] .
\end{aligned}
$$

The three theorems then follow from (1.4), (1.5).

The initial stages of extending Kotani's analysis are obvious. The proper analog 
of $h_{ \pm}$are:

$$
m_{ \pm}(\omega, E)=-u_{ \pm}( \pm 1) / u_{ \pm}(0)
$$

where $u_{ \pm}$are the solutions $1^{2}$ at $\pm \infty$. The analog of (1.2) which will come from an analog of $(1.1)$ is

$$
E\left(\operatorname{Im}\left(\left[m_{+}+m_{-}+E-V(0)\right]^{-1}\right)\right)=-\partial \gamma(E) / \partial(\operatorname{Im} E) .
$$

The analog of (1.3) is also easy:

$$
E\left(\ln \left|m_{+}\right|\right)=E\left(\ln \left|m_{-}\right|\right)=-\gamma(E) .
$$

The analog of (1.4) is more subtle because Kotani's proof does not seem to extend. However, our first proof of (1.8) was by using the idea of Delyon-Souillard [5] to use linear interpolation to force the discrete case to look like the continuum case. By a more direct proof we will show, in Sect. 2, that

$$
E\left(\ln \left[1+\left(\operatorname{Im} E / \operatorname{Im} m_{ \pm}\right)\right]\right)=2 \gamma(E) .
$$

It is not completely trivial to get an analog of (1.5). The key is the inequality

$$
\ln (1+x) \geqq x /\left(1+\frac{1}{2} x\right) .
$$

From this and (1.8), we will get, in Sect. 2, two inequalities which are close enough to the equalities (1.4), (1.5) to prove Thms. 1-3 in Sect. 3. In Sect. 4, we make a remark on the connection of these results and the work of Carmona [2].

\section{The $m$ Functions}

Given $E$ with $\operatorname{Im} E>0$ and $\omega$, it is easy to show that the difference equation

$$
u(n+1)+u(n-1)+V_{\omega}(n) u(n)=E u(n)
$$

has unique solutions $u_{ \pm}(n)$ which are $\ell^{2}$ at $\pm \infty$. Moreover,

$$
\left.\left.2 i \operatorname{Im} \overline{\left(u_{ \pm}(0)\right.} u_{ \pm}( \pm 1)\right)=\overline{u_{ \pm}(0)} u_{ \pm}( \pm 1)\right)-\overline{u_{ \pm}( \pm 1)} u_{ \pm}(0) .
$$

Recognizing this as a Wronskian of solutions of (2.1) for $E$ and $\bar{E}$, and using the fact that $u_{ \pm} \rightarrow 0$ at $\pm \infty$, one finds that

$$
\left.\operatorname{Im} \overline{\left(-u_{ \pm}(0)\right.} u_{ \pm}( \pm 1)\right)=\operatorname{Im} E\left(\sum_{j=1}^{\infty}\left|u_{ \pm}( \pm j)\right|^{2}\right)
$$

so that $u_{ \pm}(0) \neq 0$, and we can define

$$
m_{ \pm}(\omega, E)=-\frac{u_{ \pm}( \pm 1)}{u_{ \pm}(0)}
$$

and by (2.2), it obeys $\operatorname{Im} m_{ \pm}>0$. For later purpose we note that

$$
m_{ \pm}\left(T^{-n} \omega\right)=-u_{ \pm}(n \pm 1) / u_{ \pm}(n)
$$

so that the equation of motion for $u$ yields

$$
m_{ \pm}\left(T^{-n} \omega\right)=V(n)-E-\left[m_{ \pm}\left(T^{-n \pm 1} \omega\right)\right]^{-1},
$$


and in particular

$$
\frac{u_{-}(1)}{u_{-}(0)}=m_{-}+E-V(0) .
$$

As usual, $\left(H_{\omega}-E\right)^{-1}$ has an integral kernel $G_{\omega}(n, m ; E)$ which is symmetric in $n, m$ and for $n \leqq m$ :

$$
G_{\omega}(n, m ; E)=u_{-}(n) u_{+}(m) /\left[u_{+}(1) u_{-}(0)-u_{-}(1) u_{+}(0)\right] .
$$

In particular, (2.3) and (2.6) yield

$$
-G_{\omega}(0,0 ; E)^{-1}=m_{+}+m_{-}+E-V(0) .
$$

Now, $G_{\omega}(0,0 ; E)$ is related to the density of states by $[1,8]$

$$
E\left(G_{\omega}(0,0 ; E)\right)=\int \frac{d k\left(E^{\prime}\right)}{E^{\prime}-E} .
$$

The Thouless formula [1] says that

$$
\gamma(E)=\int \ln \left|E-E^{\prime}\right| d k\left(E^{\prime}\right)
$$

Equations (2.7), (2.8) and (2.9) immediately imply:

Proposition 2.1. $E\left(\operatorname{Im}\left(\left[m_{+}+m_{-}+E-V_{\omega}(0)\right]^{-1}\right)\right)=-\partial \gamma(E) / \partial(\operatorname{Im} E)$.

We let $H_{\omega}^{+}$be the operator on $\ell_{2}(1, \infty)$ which is obtained from $H_{\omega}$ by imposing the boundary condition (bc) $u(0)=0$. If $w(n)$ obeys $(2.1)$ with the bc $w(0)=0$, $w(1)=1$, then for $n \leqq m$ :

$$
\left(H_{\omega}^{+}-E\right)^{-1}(n, m)=w(n) u_{+}(m) /\left[u_{+}(1) w_{+}(0)-w(1) u_{+}(0)\right],
$$

and in particular

$$
m_{+}(\omega, E)=\left(H_{\omega}^{+}-E\right)^{-1}(1,1)
$$

By the spectral theorem, the right side of (2.10) has the form

$$
\int \frac{d \rho(x)}{x-E}
$$

where $\int d \rho \equiv 1$ and $\rho$ is supported on $\left[-\|f\|_{\infty}-2,\|f\|_{\infty}+2\right]$. From this representation one easily obtains an upper bound on $\left|m_{+}\right|$and a lower bound on $\left|\mathrm{Im} m_{+}\right|$and so:

Proposition 2.2. For any fixed $E$ with $\operatorname{Im} E>0$, there are constants $c_{1}(E), c_{2}(E), d_{1}(E)$, $d_{2}(E)$ in $(0, \infty)$ with

$$
\begin{aligned}
& c_{1}(E) \leqq\left|m_{+}(\omega, E)\right| \leqq c_{2}(E), \\
& d_{1}(E) \leqq \operatorname{Im} m_{+}(\omega, E) \leqq d_{2}(E),
\end{aligned}
$$

for all $\omega$.

From the bounds on $\left|m_{+}\right|$and the fact that for a.e. $\omega$ every solution either 
"decays" as $e^{-\gamma|n|}$ or grows as $e^{+\gamma|n|}$, we see that

$$
\lim _{n \rightarrow \infty} \frac{1}{n} \ln \left|u_{+}(n) / u_{+}(0)\right|=-\gamma
$$

Since $\ln \left|u_{+}(n) / u_{+}(0)\right|=\sum_{j=0}^{n-1} \ln \left|m_{+}\left(T^{-j} \omega\right)\right|$ (by (2.4)), we can apply the individual ergodic theorem $\left(\ln \left|m_{+}(\omega)\right|\right.$ is bounded and so in $L^{1}$ by Prop. 2.2) to find

Proposition 2.3. $E\left(\ln \left|m_{+}(\omega, E)\right|\right)=-\gamma(E)$.

Now, we come to the first result of this note that is essentially new.

Proposition 2.4. $E\left(\ln \left(1+\left[\operatorname{Im} E / \operatorname{Im} m_{+}(\omega, E)\right]\right)\right)=2 \gamma(E)$.

Proof. We start with (2.5). Taking imaginary parts, then dividing by $\operatorname{Im} m_{+}$and taking logs we find

$$
\ln \left(1+\left[\operatorname{Im} E / \operatorname{Im} m_{+}(\omega, E)\right]\right)=\ln \left(-\operatorname{Im}\left[m_{+}(T \omega, E)\right]^{-1}\right)-\ln \left(\operatorname{Im} m_{+}(\omega, E)\right) .
$$

But $-\operatorname{Im}\left[m_{+}^{-1}\right]=\operatorname{Im} m_{+} /\left|m_{+}\right|^{2}$, so taking expectations of both sides and using the invariance of $\mu$ under $T$, we find that the expectation of the right side is $-E\left(\ln \left|m_{+}\right|^{2}\right)$ which is $2 \gamma$ by Prop. 2.3.

Lemma 2.5. For $x \geqq 0, \log (1+x) \geqq x /\left(1+\frac{1}{2} x\right)$.

Proof. Both sides are equal at $x=0$. The derivative of the left hand side is $(1+x)^{-1}$ and that of the right is $\left(1+\frac{1}{2} x\right)^{-2}=\left(1+x+\frac{1}{4} x^{2}\right)^{-1}$, so we get the inequality by integrating.

Theorem 2.6. Let $b(\omega, E)=m_{+}+m_{-}+E-V(0)$ and $n_{ \pm}=\operatorname{Im} m_{ \pm}+\frac{1}{2} \operatorname{Im} E$. Then:

(a) $E\left(\left(n_{ \pm}\right)^{-1}\right) \leqq 2 \gamma(E) / \operatorname{Im} E$,

(b) $E\left(\left[n_{+}^{-1}+n_{-}^{-1}\right]\left\{\left(n_{+}-n_{-}\right)^{2}+(\operatorname{Re} b)^{2}\right\} /|b|^{2}\right) \leqq 4\left[(\operatorname{Im} E)^{-1} \gamma(E)-\partial \gamma(E) / \partial \operatorname{Im} E\right]$.

Proof. (a) follows immediately from Prop. 2.4 and the inequality in the lemma. To get (b), we write $\left(n_{+}-n_{-}\right)^{2}=\left(n_{+}+n\right)^{2}-4 n_{+} n_{-}$, and using the fact that $n_{+}+$ $n_{-}=\operatorname{Im} b$, we see the argument in the expectation is $n_{+}^{-1}+n_{-}^{-1}-4\left(n_{+}+n_{-}\right) / b^{2}=$ $n_{+}^{-1}+n_{-}^{-1}+4 \operatorname{Im}(1 / b)$. We use Prop. 2.1 to get $E(\operatorname{Im}(1 / b))$ and (a) to bound $E\left(n_{ \pm}^{-1}\right)$ to get the required inequalities.

\section{Proofs of the Theorems}

Given Thm. 2.6, the proof below follows the strategy of Kotani [10] with some changes of tactics. We begin by recalling without proofs some basic facts about Herglotz functions. As remarked by Kotani [10], these are proven most easily by mapping the upper half plane to the disc, taking logs and using the theory of $\mathrm{H}_{2}$ functions (see e.g. $[6,9]$ ).

(1) $F(z)$ defined in $\operatorname{Im} z>0$ is called Herglotz if it is analytic and has $\operatorname{Im} F(z)>0$ there. A typical example (indeed, up to linear factors, every example) is the Steiltjes 
transform of a measure, $\mu$, on $R$, viz:

$$
F(z)=\frac{1}{\pi} \int \frac{d \mu(x)}{x-z}
$$

(2) $\lim F(x+i \varepsilon) \equiv F(x+i 0)$ exists (and is finite and non-zero) for a.e. $x \in R$. $\varepsilon \downarrow 0$

(3) If $F$ comes from $\mu$, then $d \mu_{\text {ac }}$, the absolutely continuous part of $\mu$, obeys

$$
d \mu_{\mathrm{ac}}(x)=[\operatorname{Im} F(x+i 0)] d x .
$$

(4) If $F$ comes from $\mu, d \mu_{\mathrm{sing}} \equiv d \mu-d \mu_{\mathrm{ac}}$ is supported on $\left\{x \mid \lim _{\varepsilon \downarrow 0} \operatorname{Im} F(x+i \varepsilon)\right.$ $=\infty\}$.

(5) If $F(x+i 0)=G(x+i 0)$ for $x \in A$, a set with positive Lebesgue measure and $F$ and $G$ are Herglotz, then $F=G$.

(6) If $\operatorname{Re} F(x+i 0)=0$ a.e. $x \in I$, an open interval, then $F$ has an analytic continuation through $I$ and $F(x+i 0) \neq 0$ for any $x$ in $I$.

(7) By (4) and (6), if $F$ is a Steiltjes transform and $\operatorname{Re} F(x+i 0)=0$ on $I$, then $\mu=\mu_{\mathrm{ac}}$ on $I$.

Proof of Theorem 1. By (2.8), (2.9), $-\gamma(E)$ is the real part of a function whose derivative $\int\left(d h\left(E^{\prime}\right) / E^{\prime}-E\right)$ is a Steiltjes transform. Thus, by (2) above, $\lim d \gamma\left(E^{0}+i \varepsilon\right) / d \varepsilon$ exists for a.e. $E_{0}$. For any such $E_{0}$ where also $\gamma\left(E_{0}\right)=0$, we have $\varepsilon \downarrow 0$ that

$$
\lim _{\varepsilon \downarrow 0} \gamma\left(E_{0}+i \varepsilon\right) / \varepsilon=\lim _{\varepsilon \downarrow 0} d \gamma\left(E_{0}+i \varepsilon\right) / d \varepsilon,
$$

and in particular the limit is finite. Thus, by Thm. 2.6(a),

$$
\varlimsup_{\varepsilon \downarrow 0} E\left(\frac{1}{\operatorname{Im} m_{ \pm}\left(\omega, E_{0}+i \varepsilon\right)}\right)<\infty .
$$

By $(2.10 \mathrm{~b})$, for every $\omega, m_{ \pm}(\omega, E+i 0)$ exists for a.e. $E$ so for a.e. $E, m_{ \pm}(\omega, E+i 0)$ exists for a.e. $\omega$. Thus, for a.e. $E_{0}$ for which $\gamma\left(E_{0}\right)=0$, we have by (3.4) and Fatou's lemma that

$$
E\left(\frac{1}{\operatorname{Im} m_{ \pm}\left(\omega, E_{0}+i 0\right)}\right)<\infty .
$$

So, for a.e. $\omega, E_{0}, \operatorname{Im} m_{ \pm}\left(\omega, E_{0}+i 0\right)>0$. Since $m_{+}+m_{-}+E-V(0)$ has a finite limit for a.e. $\omega, E, \operatorname{Im} G>0$ a.e. $E_{0}, \omega$ which implies $\mu_{\mathrm{ac}}$ has a positive component on such $E_{0}$ by (3.2).

Proof of Theorem 2. By (3.3), (3.5) and Thm. 2.6(b) and Fatou again, we learn that, for a.e. pair $\{(\omega, E) \mid \gamma(E)=0\}$, we have that

$$
\begin{gathered}
\operatorname{Im} m_{+}\left(\omega, E_{0}+i 0\right)=\operatorname{Im} m_{-}\left(\omega, E_{0}+i 0\right), \\
\operatorname{Re}\left(m_{+}+m_{-}+E_{0}-V(0)\right)\left(\omega, E_{0}+i 0\right)=0 .
\end{gathered}
$$


By (6) above, $m_{+}+m_{-}+E-V(0)$ is analytic on $I$ and nonzero, so (by (2.7)) $G$ is analytic through $I$ which, by (4) above, implies $d \mu_{\text {sing }}=0$ on $I$.

Proof of Theorem 3. Suppose that $\gamma(E)=0$ on a set $A$ with positive measure. Suppose we know $V_{\omega}(n)$ on $n \leqq 0$. Then, $\left\{V_{\omega}(n)\right\}_{n \leqq-1}$ determines $m_{-}$and so by (3.6), (3.7), $m_{+}$is determined for a.e. $E_{0} \in A$ (and a.e. $\left.\omega\right)$ by $\left\{V_{\omega}(n)\right\}_{n \leqq 0}$ and then by (5) above, $m_{+}$is determined for all $E$. Thus the lemma below (which we learned from P. Deift) shows that $\left\{V_{\omega}(n)\right\}_{n \leqq 0}$ determines $\left\{V_{\omega}(n)\right\}_{n \geqq 1}$.

Lemma 3.1. $\left\{V_{\omega}(n)\right\}_{n \geqq 1}$ can be constructed from $m_{+}(\omega, E)$.

Proof. By $(2.10), m_{+}(\omega, E)$ determines $\left(H_{\omega}^{+}\right)^{k}(1,1)$. But it is easy to see that $\left(H_{\omega}^{+}\right)^{2 k+1}(1,1)=V_{\omega}(k+1)+a$ function of $\left\{V_{\omega}(j)\right\}_{1 \leqq j \leqq k}$, so that inductively $\left(H_{\omega}^{+}\right)^{k}(1,1)$ determines $V_{\omega}(j)$.

\section{A Connection with some work of Carmona}

In [2], Carmona proved an interesting deterministic theorem showing that certain conditions on $\{V(n)\}_{n \geqq 0}$ imply $H=H_{0}+V$ has only absolutely continuous spectrum in some interval. Here we give another condition which is clearly closely connected to his which yields the same conclusion. For any $V$ yielding a limit point situation at $\pm \infty$, say $|V(n)| \geqq-C n^{2}$, we still have functions $m^{ \pm}(E)$ and $m^{ \pm}$depend only on $\{V(n)\}_{ \pm n \geqq 1}$.

Theorem 4.1. If $\lim _{\varepsilon \downarrow 0} \operatorname{Imm}^{+}(E+i \varepsilon)>0$ for all $E$ in a set $A$, then for the spectral measure $d \mu$ associated to $\delta_{0}$, we have $\mu_{\text {sing }}(A)=0$.

Proof. By assertion (4) above (the theorem of de'Vallee Poussin), $\mu_{\text {sing }}(C)=0$, where $C=\left\{E\left|\overline{\lim _{\varepsilon \downarrow 0}}\right| G(0,0 ; E+i \varepsilon) \mid<\infty\right\}$. But since $G=-\left(m_{+}+m_{-}+E-V(0)\right)^{-1}$, we have that $|G| \leqq\left(\operatorname{Im} m_{+}+\operatorname{Im} m_{-}+\operatorname{Im} E\right)^{-1} \leqq\left(\operatorname{Im} m^{+}\right)^{-1}$ so the hypothesis implies $A \subset C$.

This is connected to the considerations of Kotani, in that:

Proposition 4.2. In the stochastic context of Sect. $1-3$, if $\gamma(E)=0$ on an interval, $I$, then for a.e. $\omega, \operatorname{Im}^{+}(E+i 0, \omega)>0$ for all $E \in I$.

Proof. As we saw in Sect. 3, $\operatorname{Im}\left(m_{+}+m_{-}\right)$is everywhere nonzero and $\operatorname{Im} m_{+}=$ $\frac{1}{2} \operatorname{Im}\left(m_{+}+m_{-}\right)$.

This shows that the periodic example of Carmona [2] can be analyzed using Thm. 4.1. Similarly, these methods extend to the continuum case and it must be true that for the Stark problem $-d^{2} / d x^{2}-x, \operatorname{Im} m^{+}>0$ for all $E$. This leaves us with an open question: Within the stochastic setting, if $\gamma(E)=0$ for all $E \in I$, is it true that for all $\omega$ and every compact $K \subset I$, we have that $\sup _{E \subset K, x>0}\left\|U_{E}(x, 0)\right\|<\infty$, where $U_{E}(x, 0)$ is the transfer matrix from 0 to $x$ ? 
Acknowledgement. It is a pleasure to thank S. Kotani for making his preprint available, and W. Craig and P. Deift for useful discussions.

\section{References}

1. Avron, J., Simon, B.: Almost periodic Schrodinger operators, II. The integrated density of states. Duke Math. J. (to appear)

2. Carmona, R.: One dimensional Schrondinger operators with random or deterministic potentials: New spectral types. J. Funct. Anal. 51, (1983)

3. Casher, A., Lebowitz, J.: Heat flow in disordered harmonic chains. J. Math. Phys. 12, 8 (1971)

4. Craig, W., Simon, B.: Subharmonicity of the Lyaponov index. Duke Math. J. (submitted)

5. Delyon, F., Souillard, B.: The rotation number for finite difference operators and its properties. Commun. Math. Phys. (to appear)

6. Donoghue, W.: Distributions and Fourier transforms. New York: Academic Press 1969

7. Ishii, K. : Localization of eigenstates and transport phenomena in the one dimensional disordered system. Supp. Theor. Phys. 53, 77-138 (1973)

8. Johnson, R., Moser, J. : The rotation number for almost periodic potentials. Commun. Math. Phys. 84, 403-438 (1982)

9. Katznelson, Y.: An introduction to harmonic analysis. New York: Dover 1976

10. Kotani, S.: Lyaponov indices determine absolutely continuous spectra of stationary random onedimensional Schrondinger operators. Proc. Kyoto Stoch. Conf., 1982

11. Pastur, L.: Spectral properties of disordered systems in the one body approximation. Commun. Math. Phys. 75, 179-196 (1980)

Communicated by T. Spencer

Received December 15, 1982 